\title{
The regularity of powers of edge ideals
}

\author{
Arindam Banerjee
}

Received: 3 September 2013 / Accepted: 30 May 2014 / Published online: 25 June 2014

(C) Springer Science+Business Media New York 2014

\begin{abstract}
In this paper, we prove the existence of a special order on the set of minimal monomial generators of powers of edge ideals of arbitrary graphs. Using this order, we find new upper bounds on the regularity of powers of edge ideals of graphs whose complement does not have any induced four cycles.
\end{abstract}

Keywords Castelnuovo-Mumford regularity $\cdot$ Powers of edge ideals $\cdot$ Cricket free

Mathematics Subject Classification (2000) Primary 13-02 · 13F55 - 05C10

\section{Introduction}

In this work, we find new upper bounds for the regularity of some classes of monomial ideals associated to graphs. Our original motivation is the following question, which is the base case of the Open Problem 1.11(2) in [13]:

Question 1.1 Let $I(G)$ be the edge ideal of a graph $G$ which does not have any induced four cycle in its complement. If $\operatorname{reg}(I(G)) \leq 3$, then is it true that for all $s \geq 2, I(G)^{s}$ has linear minimal free resolution?

Bounds on the regularity of edge ideals have been studied by a number of researchers [1-9,11-13]. For example, Fröberg [3] has shown that if when $I(G)$ is the edge ideal of a graph whose complement does not have any induced cycle of size greater than or equal to 4 , then $I(G)$ has linear minimal free resolution.

We are interested in finding upper bounds on the regularities of the higher powers of $I(G)$. Herzog et al. have shown in [6] that if $I(G)$ is the edge ideal of a graph $G$ which

\footnotetext{
A. Banerjee $(\varangle)$

Department of Mathematics, University of Virginia, Charlottesville, VA, USA

e-mail: ab4cb@virginia.edu
} 
has no induced cycle of length $\geq 4$ in its complement (that is $I(G)$ has linear minimal free resolution), then for all $s \geq 2, I(G)^{s}$ has linear minimal free resolution. Fransisco, Hà, and Van-Tuyl have further shown that if $I(G)^{s}$ has linear minimal free resolution for some $s$, then $G$ has no induced four cycle in its complement [13, Proposition 1.8]. These two results lead us to study bounds on the regularity of powers of $I(G)$ when $G$ has no induced four cycle in its complement. Our main result is Theorem 6.17 where we prove that all higher powers of edge ideals of a gap-free (equivalently, no induced four cycle in complement, as observed in Sect. 2) and cricket-free (defined in Sect. 2) graph have linear minimal free resolution. More precisely:

Theorem 1.2 For every gap-free and cricket-free graph $G$ and for all $s \geq 2$, $\operatorname{reg}\left(I(G)^{S}\right)=2 s$. As a consequence, $I(G)^{s}$ has a linear minimal free resolution.

This partially answers Question 1.1, as we prove in Sect. 3 that edge ideals of gap-free and cricket-free graphs have regularity less than or equal to 3 (Theorem 3.4). As claw free graphs (defined in Sect. 2) are automatically cricket free, our results generalize a previous result by Nevo [12, Theorem 1.2] which states that the edge ideals of gap-free and claw-free graphs have regularity less than or equal to 3 , and their squares have linear minimal free resolutions.

In order to prove Theorem 6.17, we first show that the minimal monomial generators of powers of the edge ideal $I(G)$ of any finite simple graph $G$ have a specific order with a nice property (Lemma 4.11, Theorem 4.12). More precisely:

Theorem 1.3 For each $n \geq 1$, there exists an ordered list $L^{(n)}$ of minimal monomial generators of $I(G)^{n}$ which has the following property:

For all $k \geq 1$ and for all $j \leq k$, if $\left(L_{j}^{(n)}: L_{k+1}^{(n)}\right)$ is not contained in $\left(I(G)^{n+1}: L_{k+1}^{(n)}\right)$; then there exists $i \leq k$ such that $\left(L_{i}^{(n)}: L_{k+1}^{(n)}\right)$ is generated by a variable and $\left(L_{j}^{(n)}\right.$ : $\left.L_{k+1}^{(n)}\right) \subseteq\left(L_{i}^{(n)}: L_{k+1}^{(n)}\right)$. For monomials $m$ and $n,(m: n)$ stands for $((m):(n))$.

Using this ordering, we shall prove that $\operatorname{reg}\left(I(G)^{n}\right)$ is bounded above by the maximum of $\operatorname{reg}\left(I(G)^{n}: e_{1} \ldots e_{n-1}\right)+2 n-2$ for all possible $(n-1)$-fold products of edges $e_{1} \ldots e_{n-1}$ and $\operatorname{reg}\left(I(G)^{n-1}\right)$ (see Theorem 5.2). Next, we prove that the ideals $\left(I(G)^{n}: e_{1} \ldots e_{n-1}\right)$ are quadratic monomial ideals with generators satisfying certain conditions (see Theorems 6.1, 6.5, 6.7). Finally, using polarization, we get edge ideals corresponding to these quadratic monomial ideals with some regularity (see [8], Sect. 3.2 and Exercise 3.15 of [10] for details) and using Fröberg's theorem (see Theorem 1 of [3] and Theorem 1.1 of [13]) we get bounds on them. As a consequence, we also get a different proof of the Herzog et al.'s result mentioned above (Theorem 6.16).

\section{Preliminaries}

Throughout this paper, we let $G$ be a finite simple graph with vertex set $V(G)$. For $u, v \in V(G)$, we let $d(u, v)$ denote the distance between $u$ and $v$, the fewest number of edges that must be traversed to travel from $u$ to $v$.

A subgraph $G^{\prime} \subseteq G$ is called induced if $u v$ is an edge of $G^{\prime}$ whenever $u$ and $v$ are vertices of $G^{\prime}$, and $u v$ is an edge of $G$. 
The complement of a graph $G$, for which we write $G^{c}$, is the graph on the same vertex set in which $u v$ is an edge of $G^{c}$ if and only if it is not an edge of $G$.

Finally, we denote by $C_{k}$ the cycle on $k$ vertices and by $K_{m, n}$ the complete bipartite graph with $m$ vertices on one side, and $n$ on the other.

Definition 2.1 Let $G$ be a graph. We say that two disjoint edges $u v$ and $x y$ form a gap in $G$ if $G$ does not have an edge with one endpoint in $\{u, v\}$ and the other in $\{x, y\}$. A graph without gaps is called gap free. Equivalently, $G$ is gap free if and only if $G^{c}$ contains no induced $C_{4}$.

Thus, $G$ is gap free if and only if it does not contain two vertex-disjoint edges as an induced subgraph.

Definition 2.2 Any graph isomorphic to $K_{1,3}$ is called a claw. Any graph isomorphic to $K_{1, n}$ is called an $n$-claw. If $n>1$, then the vertex with degree $n$ is called the root in $K_{1, n}$. A graph without an induced claw is called claw free. A graph without an induced $n$-claw is called $n$-claw free.

Definition 2.3 Any graph isomorphic to the graph with set of vertices $\left\{w_{1}, w_{2}, w_{3}\right.$, $\left.w_{4}, w_{5}\right\}$ and set of edges $\left\{w_{1} w_{3}, w_{2} w_{3}, w_{3} w_{4}, w_{3} w_{5}, w_{4} w_{5}\right\}$ is called a cricket. A graph without an induced cricket is called cricket free.

Definition 2.4 An edge in a graph is called a whisker if each of its vertices has degree one.

Definition 2.5 A graph is called an anticycle if its complement is a cycle.

Observation 2.6 A claw free graph is cricket free.

If $G$ is a graph without isolated vertices then let $S$ denote the polynomial ring on the vertices of $G$ over some fixed field $K$. Recall that the edge ideal of $G$ is

$$
I(G)=(x y: x y \text { is an edge of } G) .
$$

Definition 2.7 Let $S$ be a standard graded polynomial ring over a field $K$. The Castelnuovo-Mumford regularity of a finitely generated graded $S$ module $M$, written $\operatorname{reg}(M)$, is given by

$$
\operatorname{reg}(M):=\max \left\{j-i \mid \operatorname{Tor}_{i}(M, K)_{j} \neq 0\right\} .
$$

Definition 2.8 We say that $I(G)^{s}$ is $k$-steps linear whenever the minimal free resolution of $I(G)^{s}$ over the polynomial ring is linear for $k$ steps, i.e., $\operatorname{Tor}_{i}^{S}\left(I(G)^{s}, K\right)_{j}=0$ for all $1 \leq i \leq k$ and all $j \neq i+2 s$. We say that $I(G)$ has linear minimal free resolution if the minimal free resolution is $k$-steps linear for all $k \geq 1$.

We end this section by recalling a few well-known results; see [1] and [13].

Observation 2.9 Let $I(G)$ be the edge ideal of a graph $G$. Then, $I(G)^{s}$ has linear minimal free resolution if and only if $\operatorname{reg}\left(I(G)^{s}\right)=2 s$. 
Lemma 2.10 Let $I \subseteq S$ be a monomial ideal. Then, for any variable $x, \operatorname{reg}(I, x) \leq$ $\operatorname{reg}(I)$. In particular, if $v$ is a vertex in a graph $G$, then $\operatorname{reg}(I(G-v)) \leq \operatorname{reg}((I(G))$.

The next statement follows from Lemma 2.10 of [1]:

Lemma 2.11 Let I $\subseteq S$ be a monomial ideal, and let $m$ be a monomial of degree $d$. Then

$$
\operatorname{reg}(I) \leq \max \{\operatorname{reg}(I: m)+d, \operatorname{reg}(I, m)\}
$$

Moreover, if $m$ is a variable $x$ appearing in $I$, then $\operatorname{reg}(I)$ is equal to one of these terms.

Finally, the following theorem due to Fröberg (see Theorem 1 of [3] and Theorem 1.1 of [13]) is used repeatedly throughout this paper:

Theorem 2.12 The minimal free resolution of $I(G)$ is linear if and only if the complement graph $G^{c}$ is chordal, that is, no induced cycle in $G^{c}$ has length greater than three.

\section{Gap-free graphs}

In this section we observe some basic results concerning gap-free graphs and their regularity. We prove that a cricket free and gap free graph has regularity at most 3 , generalizing Nevo's result [1, Theorem 3.3] that a gap-free and claw-free graph has regularity at most 3 . We generalize Nevo's result in another direction by proving that $n$-claw free and gap free graphs have regularity at most $n$.

Definition 3.1 For any graph $G$, we write $\operatorname{reg}(G)$ as a shorthand for $\operatorname{reg}(I(G))$.

Recall that the star of a vertex $x$ of $G$, which we denote by st $x$, is defined as

$$
\text { stx }=\{y \in V(G): x y \text { is an edge of } G\} \cup\{x\} .
$$

The following lemma, which is Lemma 3.1 of [1], will be used repeatedly in this work.

Lemma 3.2 Let $x$ be a vertex of $G$ with neighbors $y_{1}, y_{2}, \ldots, y_{m}$. Then

$$
(I(G): x)=\left(I(G-s t x), y_{1}, \ldots, y_{m}\right) \text { and }(I(G), x)=(I(G-x), x)
$$

Thus, $\operatorname{reg}(G) \leq \max \{\operatorname{reg}(G-s t x)+1, \operatorname{reg}(G-x)\}$. Moreover, $\operatorname{reg}(G)$ is equal to one of these terms.

The next proposition is Proposition 3.2 of [1].

Proposition 3.3 Let $G$ be gap free, and let $x$ be a vertex of $G$ of highest degree. Then, $d(x, y) \leq 2$ for all vertices $y$ of $G$. 
We prove the next two theorems using Proposition 3.3. Our proof is motivated by the proof of Theorem 3.3 of [1].

Theorem 3.4 Suppose $G$ is both cricket free and gap free. Then, $\operatorname{reg}(G) \leq 3$.

Proof Let $x$ be a vertex of maximum degree. As $G$ is gap free and cricket free, so is $G-x$. By induction, $G-x$ has regularity less than or equal to 3. Because of Lemma 3.2 and Theorem 2.12, it is enough to show that $(G-\mathrm{st} x)^{c}$ has no induced cycle of length greater than or equal to 4 . As $G$ is gap free, so is $(G-\mathrm{st} x)$; hence, $(G-\mathrm{st} x)^{c}$ has no induced 4-cycle. So, it is enough to show that it does not have an induced cycle of length $\geq 5$.

Let $\left\{y_{1}, y_{2}, y_{3}, y_{4}, \ldots, y_{n}\right\}$ be an induced cycle $(n \geq 5)$ in $(G-\text { st } x)^{c}$; because of Proposition 3.3, there is a $w$ such that $x w$ and $w y_{1}$ are edges in $G$. As $y_{2} y_{n}$ is an edge in $G$, and neither $y_{1} y_{2}$ nor $y_{1} y_{n}$ is an edge in $G$, at least one of $w y_{2}$ and $w y_{n}$ is an edge in $G$. If both are edges, then $\left\{x, w, y_{1}, y_{2}, y_{n}\right\}$ forms an induced cricket.

Suppose only one of them is an edge. Without loss of generality, we may assume that $w y_{2}$ is an edge. As $y_{3} y_{n}$ is an edge in $G$, and $G$ is gap free, $w y_{3}$ is an edge in $G$; otherwise $\left\{x, w, y_{3}, y_{n}\right\}$ forms a gap in $G$. This makes $\left\{x, w, y_{1}, y_{2}, y_{3}\right\}$ an induced cricket.

Theorem 3.5 The edge ideal of a graph, which is gap free and n-claw free, has regularity less than or equal to $n$.

Proof For $n=3$, this was proved by Nevo and this is Theorem 3.3 of [1]. So we may assume $n \geq 4$. Let $x$ be a vertex with maximum degree. Because of Lemma 3.2, it is enough to show that $G-$ st $x$ has regularity less than or equal to $n-1$; as $G-x$ has regularity less than or equal to $n$ by induction on number of vertices. Hence, it is enough to show that $G-$ st $x$ is $(n-1)$-claw free.

If $a_{1}, a_{2}, a_{3}, \ldots, a_{n}$ is a $(n-1)$-claw with root $a_{1}$ in $G-$ st $x$, then any $w$ in the neighborhood of $x$ is either connected to $a_{1}$ or all of $a_{2}, a_{3}, \ldots, a_{n}$; otherwise, if $w$ is not connected to $a_{1}$ and $a_{i}$, then $x w$ and $a_{1} a_{i}$ will form a gap. If $a_{1}$ is connected to all neighbors of $x$, then it has degree larger than that of $x$, a fact which contradicts the assumption $x$ is a vertex with maximum degree. Hence, there is a neighbor $w$ which is not connected to $a_{1}$ but is connected to all of $a_{2}, a_{3}, \ldots, a_{n}$. As $x$ is not connected to any of the $a_{i} \mathrm{~s},\left\{x, w, a_{2}, a_{3}, \ldots, a_{n}\right\}$ forms an $n$-claw with root $w$, which contradicts the hypothesis.

\section{Ordering the minimal monomial generators of powers of edge ideals}

Discussion 4.1 We will denote by Mingens $(J)$ the set of minimal monomial generators of an ideal $J \subset S$. Let I be an arbitrary edge ideal and set Mingens $(I)=$ $\left\{L_{1}, L_{2}, \ldots, L_{k}\right\}$. We consider the order $L_{1}>L_{2}>\cdots>L_{k}$ on $\operatorname{Mingens}(I)$ and for every integer $n \geq 2$ we endow the set Mingens $\left(I^{n}\right)$ with the following order: we set $M>N$ for $M, N \in \operatorname{Mingens}\left(I^{n}\right)$ if there exists an expression $L_{1}^{a_{1}} L_{2}^{a_{2}} \ldots L_{k}^{a_{k}}=M$ such that for all expressions $L_{1}^{b_{1}} \ldots L_{k}^{b_{k}}=N$, we have $\left(a_{1}, \ldots, a_{k}\right)>_{\text {lex }}\left(b_{1}, \ldots, b_{k}\right)$. If $\left(a_{1}, \ldots, a_{k}\right) \geq_{\text {lex }}\left(c_{1}, \ldots, c_{k}\right)$ for all $\left(c_{1}, \ldots, c_{k}\right)$ such that $L_{1}^{c_{1}} \ldots L_{k}^{c_{k}}=M$, then 
$L_{1}^{a_{1}} L_{2}^{a_{2}} \ldots L_{k}^{a_{k}}$ is called a maximal expression of $M$. Let $L^{(n)}$ be the totally ordered set of minimal monomial generators of $I^{n}$, ordered in the way discussed above.

Definition 4.2 If $m_{1}$ is a minimal monomial generator of $I^{k}$ and $m_{2}$ is a minimal monomial generator of $I^{n}$ where $n>k$, then we say $m_{1}$ divides $m_{2}$ as an edge and use the notation $\left.m_{1}\right|^{\text {edge }} m_{2}$; if there exists $m_{3}$, a minimal monomial generator of $I^{n-k}$ with $m_{2}=m_{1} m_{3}$.

Example 4.3 If $I=(a b, b c, a d, b d)$, then $\left.a b\right|^{\text {edge }} a b^{2} d$ as $b d=\frac{a b^{2} d}{a b}$ is a minimal monomial generator of $I$ but $a b\}^{\text {edge }} a b c d$ as $c d=\frac{a b c d}{a b}$ is not a minimal monomial generator of $I$.

Discussion 4.4 We have the following for the list $L^{(n)}$ created above:

1. $L^{(1)}=L:=\left\{L_{1}>\cdots>L_{k}\right\}$

2. For any minimal monomial generator $m$ of $I^{n}, n \geq 2$, the maximal expression of $m$ is an expression of $m$ as a product of $n$ elements of $L, m=L_{i_{1}} L_{i_{2}} \ldots L_{i_{n}}$, where: a. $i_{1}$ is the minimum integer such that $\left.L_{i_{1}}\right|^{\text {edge }} m$

b. For all $l \geq 1, i_{l+1}$ is the minimal integer such that $\left.L_{i_{l+1}}\right|^{e d g e} \frac{m}{L_{i_{1}} \ldots L_{i_{l}}}$. For any edge $c d$, we say that $c d$ is a part of the maximal expression of $m$ if $c d=L_{i_{k}}$ for some $k$.

This expression is unique by the construction.

3. For two minimal monomial generators $m_{1}, m_{2}$ with maximal expressions $m_{1}=$ $L_{i_{1}} \ldots L_{i_{n}}$ and $m_{2}=L_{j_{1}} \ldots L_{j_{n}}$, we have $m_{1}>_{\text {lex }} m_{2}$ if for the minimum integer $l$ such that $i_{l} \neq j_{l}, i_{l}<j_{l}$.

4. If $L_{i}$ and $L_{j}$ are two generators of I with $i<j$, then we say " $L_{j}$ comes after $L_{i}$ " or " $L_{i}$ comes before $L_{j}$."

Example 4.5 Let $I=(a b, b c, a d, b d)$. Let $L^{(1)}=\{a b>b c>a d>b d\}$. Then $L^{(2)}=\left\{a^{2} b^{2}>a b^{2} c>a^{2} b d>a b^{2} d>b^{2} c^{2}>a b c d>b^{2} c d>a^{2} d^{2}>a b d^{2}>\right.$ $\left.b^{2} d^{2}\right\}$.

Definition 4.6 If $L_{i}=a b$ is an edge, that is a minimal monomial generator of $I$, and $m$ is a minimal monomial generator of $I^{n}, n \geq 2$, then we say that $m$ belongs to $a b$, or $m$ belongs to $L_{i}$, if $i$ is the least integer such that $\left.L_{i}\right|^{\text {edge }} m$.

Example 4.7 Let $I=(a b, b c, a d, b d)$ with $L=L^{(1)}=\{a b>b c>a d>b d\}$.

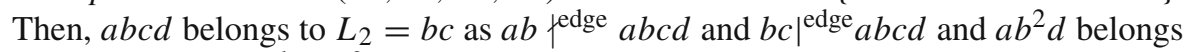
to $L_{1}=a b$ as $\left.a b\right|^{\text {edge }} a b^{2} d$.

We record several easy observations that we need in the sequel.

Observation 4.8 For two minimal monomial generators $m_{1}, m_{2}$, if $m_{1}$ belongs to an edge $L_{i}$ and $m_{2}$ belongs to another edge $L_{j}$ with $i<j$, then $m_{1}>_{\text {lex }} m_{2}$.

Observation 4.9 For two minimal monomial generators $m_{1}, m_{2}$ of $I^{n}$ which both belong to an edge $L_{i}$, we see that $m_{1}>_{l e x} m_{2}$ if and only if $\frac{m_{1}}{L_{i}}>$ lex $\frac{m_{2}}{L_{i}}$. 
Observation 4.10 Suppose $m$ is a minimal monomial generator of $I^{n}, n \geq 2$, and $g h$ is an edge which is a part of the maximal expression of $m$. Write $m=g \mathrm{hm}^{\prime}$. For any minimal monomial generator $m^{\prime \prime}$ of $I^{n-1}$ such that $m^{\prime \prime}>_{\text {lex }} m^{\prime}$, then $g h m^{\prime \prime}>_{l e x} m$.

Proof Let $L=\left\{L_{1}>L_{2}>\cdots>L_{k}\right\}$. Let $g h=L_{j}$ for some $j$. Let $m^{\prime \prime}=$ $L_{1}^{a_{1}} L_{2}^{a_{2}} \ldots L_{k}^{a_{k}}$ be the maximal expression of $m^{\prime \prime}$ and $m^{\prime}=L_{1}^{b_{1}} L_{2}^{b_{2}} \ldots L_{k}^{b_{k}}$ be the maximal expression of $m^{\prime}$. As $g h$ is a part of the maximal expression of $m$, the maximal expression of $m$ is $L_{1}^{b_{1}} \ldots L_{j}^{b_{j}+1} \ldots L_{k}^{b_{k}}$. As by assumption $\left(a_{1}, \ldots, a_{j}, \ldots, a_{k}\right)>_{\text {lex }}$ $\left(b_{1}, \ldots, b_{j}, \ldots, b_{k}\right)$, we have $\left(a_{1}, \ldots, a_{j}+1, \ldots, a_{k}\right)>_{\operatorname{lex}}\left(b_{1}, \ldots, b_{j}+1, \ldots, b_{k}\right)$. Now $L_{1}^{a_{1}} \ldots L_{j}^{a_{j}+1} \ldots L_{k}^{a_{k}}$ is an expression for $g h m^{\prime \prime}$. Hence, $g h m^{\prime \prime}>_{\text {lex }} g h m^{\prime}=m$.

The next lemma is the most important technical result of this paper as it allows us to build the framework of Sect. 5. Using the framework of Sect. 5, we obtain our bounds in Sect. 6.

Lemma 4.11 For all $k \geq 1$ and for all $j \leq k$, if $\left(L_{j}^{(n)}: L_{k+1}^{(n)}\right)$ is not contained in $\left(I^{n+1}: L_{k+1}^{(n)}\right)$ and $L_{j}^{(n)}$ belongs to an edge that comes before the edge $L_{k+1}^{(n)}$ belongs to, then there exists $i \leq k$, such that $\left(L_{i}^{(n)}: L_{k+1}^{(n)}\right)$ is generated by a variable, $\left(L_{j}^{(n)}: L_{k+1}^{(n)}\right) \subseteq\left(L_{i}^{(n)}: L_{k+1}^{(n)}\right)$ and $L_{i}^{(n)}$ belongs to an edge that comes before or equal to the edge $L_{j}^{(n)}$ belongs to.

Proof We proceed by induction on $n$. We recall that for two monomials $m_{1}$ and $m_{2}$, $\left(m_{1}: m_{2}\right)=\left(\frac{m_{1}}{\operatorname{gcd}\left(m_{1}, m_{2}\right)}\right)$. This is going to be used in several places.

If $n=1,\left(L_{j}: L_{k+1}\right)$ is either $\left(L_{j}\right)$, in which case $\left(L_{j}: L_{k+1}\right) \subseteq\left(I^{2}: L_{k+1}\right)$ or it is generated by a variable in which case we take $L_{i}=L_{j}$. Hence, the lemma is true for $n=1$.

Suppose the result is true for $n-1$. Let $L_{j}^{(n)}$ belong to $a b$, so that $L_{j}^{(n)}=a b M_{1}$ where $M_{1} \in L^{(n-1)}$. By assumption, $L_{k+1}^{(n)}$ belongs to an edge which comes after $a b$ in $L$. If neither $a$ nor $b$ divide $L_{k+1}^{(n)}$, then $\left(L_{j}^{(n)}: L_{k+1}^{(n)}\right) \subseteq(a b) \subseteq\left(I^{n+1}: L_{k+1}^{(n)}\right)$ which is contrary to our assumption.

Without loss of generality, we assume $a \mid L_{k+1}^{(n)}$. As $L_{k+1}^{(n)}$ is a product of edges, there exists an edge $a c$ with $\left.a c\right|^{\text {edge }} L_{k+1}$, where $a c$ is a part of the maximal expression of $L_{k+1}^{(n)}$. So, $L_{k+1}^{(n)}=a c M_{2}$ for some $M_{2} \in L^{(n-1)}$ which is the remaining part of the maximal expression. Now $a b \jmath^{\text {edge }} L_{k+1}^{(n)}$ as $L_{k+1}^{(n)}$ belongs to an edge that comes after $a b$. Hence, $b \neq c$.

If $\left(L_{j}^{(n)}: L_{k+1}^{(n)}\right) \subseteq(b)$, then we take $L_{i}^{(n)}=a b M_{2}$. Clearly, $L_{i}^{(n)}$ belongs to $a b$ or some edge that comes before $a b$. Also, $\left(L_{i}^{(n)}: L_{k+1}^{(n)}\right)=\left(a b M_{2}: a c M_{2}\right)=(b)$. Hence, $L_{i}^{(n)}$ has all the required properties.

If $\left(L_{j}^{(n)}: L_{k+1}^{(n)}\right)$ is not contained in $(b)$, then there is a variable $d$ such that $b d$ is an edge and $\left.b d\right|^{\text {edge }} M_{2}$ and $b d$ is a part of maximal expression of $M_{2}$. Let $\left(L_{j}^{(n)}\right.$ : $\left.L_{k+1}^{(n)}\right) \subseteq(f)$ where $f$ is a variable. If $\left(L_{j}^{(n)}: L_{k+1}^{(n)}\right)=(f)$, then we take $L_{i}^{(n)}=L_{j}^{(n)}$. This has all the required properties. 
So let us assume $\left(L_{j}^{(n)}: L_{k+1}^{(n)}\right)=\left(M_{1} b: M_{2} c\right) \subsetneq(f)$. Let $\left(L_{j}^{(n)}: L_{k+1}^{(n)}\right)=(f m)$ where $m$ is a monomial which is not 1 . So there is an edge $f g$ such that $\left.f g\right|^{\text {edge }} M_{1}$ and $f g$ is part of the maximal expression of $M_{1}$. If $g \nmid M_{2} c$, then $\left(L_{j}^{(n)}: L_{k+1}^{(n)}\right) \subseteq$ $(f g) \subseteq\left(I^{n+1}: L_{k+1}^{(n)}\right)$ which contradicts our assumption. So $g \mid M_{2} c$.

If $g=c$, then either $f=d$, that is $f c a b=b d a c$ or $(f c a b: b d a c)=(f)$. In the first case, $L_{k+1}=a c M_{2}=a c b d \frac{M_{2}}{b d}=f c a b \frac{M_{2}}{b d}$. Now $\left.b d\right|^{\text {edge }} M_{2}$, so $\left.a b\right|^{\text {edge }} L_{k+1}^{(n)}$ which is a contradiction. In the second case, we take $L_{i}^{(n)}=(f c)(a b) \frac{L_{k+1}^{(n)}}{b d a c}$. Clearly, $L_{i}^{(n)}$ belongs to $a b$ or a some edge that comes before $a b$ and $\left(L_{i}^{(n)}: L_{k+1}^{(n)}\right)=(f)$, which contains $\left(L_{j}^{(n)}: L_{k+1}^{(n)}\right)$. Hence, $L_{i}^{(n)}$ has the required properties.

Now let us assume $g \neq c$. So, there is an edge $g h$ such that $\left.g h\right|^{\text {edge }} M_{2}$, such that $g h$ is a part of the maximal expression of $M_{2}$. Let $\frac{M_{1}}{f g}=N_{1}$ and $\frac{M_{2}}{g h}=N_{2}$. As $\left(L_{j}^{(n)}: L_{k+1}^{(n)}\right)=(f m), f g a b N_{1} \mid f m g h a c N_{2}$. So $a b N_{1} \mid h m a c N_{2}$. So $(h m) \subset$ $\left(a b N_{1}: a c N_{2}\right)$. We observe that $\left(a b N_{1}: a c N_{2}\right)$ is either $(m)$ or $(h m)$. For if $m^{\prime} \mid m$, then $a b N_{1} \mid h m^{\prime} a c N_{2}$ implies $f g a b N_{1} \mid f m^{\prime} g h a c N_{2}$ implies $f m \mid f m^{\prime}$ implies $m=m^{\prime}$.

If $\left(N_{1} a b: N_{2} a c\right)=(m)$, then $\left(L_{j}^{(n)}: L_{k+1}^{(n)}\right) \subseteq(m)=\left(a b N_{1}: a c N_{2}\right)$. Now both $a b N_{1}$ and $a c N_{2}$ are in $L^{(n-1)}$. As $a b N_{1}$ belongs to $a b$ and $a c N_{2}$ belongs to some edge which comes after $a b, a b N_{1}>_{\text {lex }} a c N_{2}$. By induction, either $\left(a b N_{1}: a c N_{2}\right) \subseteq\left(I^{n}\right.$ : $\left.a c N_{2}\right)$ or there exists $M_{0}$ in $L^{(n-1)}, M_{0}>_{\text {lex }} a c N_{2},\left(a b N_{1}: a c N_{2}\right) \subseteq\left(M_{0}: a c N_{2}\right)$, $\left(M_{0}: a c N_{2}\right)$ is generated by a variable, and $M_{0}$ belongs to an edge that comes before or equal to $a b$. In the first case, $\left(L_{j}^{(n)}: L_{k+1}^{(n)}\right) \subseteq\left(a b N_{1}: a c N_{2}\right) \subseteq\left(I^{n}: a c N_{2}\right) \subset$ $\left(I^{n+1}: \operatorname{ghacN}_{2}\right)=\left(I^{n+1}: L_{k+1}^{(n)}\right)$, which is a contradiction. In the second case, write $L_{i}^{(n)}=g h M_{0}$. We know that $L_{i}^{(n)}>_{\operatorname{lex}} L_{k+1}^{(n)}$ as $M_{0}$ belongs to an edge that comes before or equal to $a b$. Also, $\left(L_{i}^{(n)}: L_{k+1}^{(n)}\right)=\left(M_{0}: a c N_{2}\right),\left(L_{j}^{(n)}: L_{k+1}^{(n)}\right) \subseteq(m)=$ $\left(a b N_{1}: a c N_{2}\right) \subseteq\left(M_{0}: a c N_{2}\right)$ and $\left(M_{0}: a c N_{2}\right)$ is generated by a variable.

Now let us assume $\left(a b N_{1}: a c N_{2}\right)=(\mathrm{hm})$. As $a b N_{1}>_{\text {lex }} a c N_{2}$, by induction either $\left(a b N_{1}: a c N_{2}\right) \subseteq\left(I^{n}: a c N_{2}\right)$ or there exists $M_{0}^{\prime}$ in $L^{(n-1)}, M_{0}^{\prime}>_{\text {lex }} a c N_{2}$, with $\left(a b N_{1}: a c N_{2}\right) \subseteq\left(M_{0}^{\prime}: a c N_{2}\right),\left(M_{0}^{\prime}: a c N_{2}\right)$ is generated by a variable, and $M_{0}^{\prime}$ belongs to an edge that comes before or equal to $a b$. In the first case $\operatorname{hmacN}_{2} \in I^{n}$, so $f m g h a c N_{2}=\operatorname{fgmhac} N_{2} \in I^{n+1}$. So $\left(L_{j}^{(n)}: L_{k+1}^{(n)}\right) \subseteq\left(I^{n+1}: L_{k+1}^{(n)}\right)$, which is a contradiction. In the second case, if $\left(M_{0}^{\prime}: a c N_{2}\right) \neq(h)$, then let $L_{i}^{(n)}=g h M_{0}^{\prime}$. As $M_{0}^{\prime}$ belongs to an edge that comes before or equal to $a b, L_{i}^{(n)}>_{\operatorname{lex}} L_{k+1}^{(n)}$. Also $\left(L_{i}^{(n)}\right.$ : $\left.L_{k+1}^{(n)}\right)=\left(M_{0}^{\prime}: a c N_{2}\right)$ which contains $\left(L_{j}^{(n)}: L_{k+1}^{(n)}\right)$ and is generated by a variable. If $\left(M_{0}^{\prime}: a c N_{2}\right)=(h)$ we take $L_{i}^{(n)}=f g M_{0}^{\prime}$. By same reasoning, $L_{i}^{(n)}>_{\operatorname{lex}} L_{k+1}^{(n)}$. As $L_{i}^{(n)}$ cannot be same as $L_{k+1}^{(n)}$, we observe $\left(L_{i}^{(n)}: L_{k+1}^{(n)}\right)=(f)$. So, this $L_{i}^{(n)}$ has all the required properties. This completes the proof.

Theorem 4.12 For all $k \geq 1$ and for all $j \leq k$, if $\left(L_{j}^{(n)}: L_{k+1}^{(n)}\right)$ is not contained in $\left(I^{n+1}: L_{k+1}^{(n)}\right)$, then there exists $i \leq k$, such that $\left(L_{i}^{(n)}: L_{k+1}^{(n)}\right)$ is generated by a variable and $\left(L_{j}^{(n)}: L_{k+1}^{(n)}\right) \subseteq\left(L_{i}^{(n)}: L_{k+1}^{(n)}\right)$. 
Proof We have $L_{j}^{(n)}=m m_{1}$ and $L_{k+1}^{(n)}=m m_{2}$ where $m \in \operatorname{Mingens}\left(I^{k}\right)$ and $m_{1}, m_{2} \in$ Mingens $\left(I^{n-k}\right)$ with $m_{1}$ belong to an edge that comes strictly before the edge $m_{2}$ belongs. We observe $\left(L_{j}^{(n)}: L_{k+1}^{(n)}\right)=\left(m_{1}: m_{2}\right)$ and $\left(I^{n-k+1}: m_{2}\right) \subseteq\left(I^{n+1}: m m_{2}\right)$. With these two observations, the theorem follows from Lemma 4.11. This finishes the proof.

\section{Bounding the regularity: the framework}

In this section, we create the framework from which we shall prove our bounds. The framework is created by repeated use of Lemma 2.11. Let $I$ and $J$ be two homogeneous square-free monomial ideals in $S$ generated in degrees $n_{1}$ and $n_{2}$ respectively. Assume $J \subset I$ and that $n_{2}>n_{1}$. If the unique set of minimal monomial generators of $I$ is $\left\{m_{1}, m_{2}, \ldots, m_{k}\right\}$, then repeated use of Lemma 2.11 gives us the following lemma:

\section{Lemma 5.1 Let}

$$
\begin{aligned}
& A=\max \left\{\operatorname{reg}\left(J: m_{1}\right)+n_{1}\right\} \\
& B=\max \left\{\operatorname{reg}\left(\left(J, m_{1}, \ldots, m_{l}\right): m_{l+1}\right)+n_{1} \mid 1 \leq l \leq k-1\right\} \\
& C=\operatorname{reg}(I) .
\end{aligned}
$$

Then, $\operatorname{reg} J \leq \max \{A, B, C\}$.

Proof We consider the follwing short exact sequence:

$$
0 \longrightarrow \frac{S}{\left(J: m_{1}\right)}\left(-n_{1}\right) \stackrel{. m_{1}}{\longrightarrow} \frac{S}{J} \longrightarrow \frac{S}{\left(J, m_{1}\right)} \longrightarrow 0
$$

This gives us $\operatorname{reg}(J) \leq \max \left\{\operatorname{reg}\left(J: m_{1}\right)+n_{1}=A, \operatorname{reg}\left(J, m_{1}\right)\right\}$. Let $J_{l}:=$ $\left(\left(J, m_{1}, \ldots, m_{l-1}\right): m_{l}\right)$ for all $l \geq 2$. For all $1 \leq l \leq k-1$, we can consider the exact sequence

$$
0 \longrightarrow \frac{S}{\left(J_{l+1}\right)}\left(-n_{1}\right) \stackrel{m_{l+1}}{\longrightarrow} \frac{S}{\left(J, m_{1}, \ldots, m_{l}\right)} \longrightarrow \frac{S}{\left(J, m_{1}, \ldots, m_{l+1}\right)} \longrightarrow 0
$$

This gives us

$$
\operatorname{reg}\left(J, m_{1}, \ldots, m_{l}\right) \leq \max \left\{\operatorname{reg}\left(J_{l+1}\right)+n_{1}, \operatorname{reg}\left(J, m_{1}, \ldots, m_{l+1}\right)\right\}
$$

from which $\operatorname{reg}(J) \leq \max \{A, B, C\}$ follows.

This lemma together with Theorem 4.12 gives the next theorem which is the main result we use for finding bounds on regularity of higher powers of edge ideals.

Theorem 5.2 For any finite simple graph $G$ and any $s \geq 1$, let the set of minimal monomial generators of $I(G)^{s}$ be $\left\{m_{1}, \ldots, m_{k}\right\}$. Then

$$
\operatorname{reg}\left(I(G)^{s+1}\right) \leq \max \left\{\operatorname{reg}\left(I(G)^{s+1}: m_{l}\right)+2 s, 1 \leq l \leq k, \operatorname{reg}\left(I(G)^{s}\right)\right\} .
$$


Proof Minimal monomial generators of $I(G)^{s}$ forms the ordered list $L^{(s)}$ from Sect. 4. So, by Lemma 5.1,

$$
\operatorname{reg}\left(I(G)^{s+1}\right) \leq \max \{A, B, C\}
$$

where

$$
\begin{aligned}
& A=\max \left\{\operatorname{reg}\left(I(G)^{s+1}: L_{1}^{(s)}\right)+2 s\right\} \\
& B=\max \left\{\operatorname{reg}\left(\left(\left(I(G)^{s+1}, L_{1}^{(s)}, \ldots, L_{l}^{(s)}\right): L_{l+1}^{(s)}\right)+2 s \mid 1 \leq l \leq k-1\right\}\right. \\
& C=\operatorname{reg}\left(I(G)^{s}\right) .
\end{aligned}
$$

But in light of Theorem 4.12, $\left(\left(I(G)^{s+1}, L_{1}^{(s)}, \ldots, L_{l}^{(s)}\right): L_{l+1}^{(s)}\right)$ is the same as $\left(\left(I(G)^{s+1}: L_{l+1}^{(s)}\right)\right.$, some variables). So, by Lemma 2.10

$$
\operatorname{reg}\left(\left(I(G)^{s+1}, L_{1}^{(s)}, \ldots, L_{l}^{(s)}\right): L_{l+1}^{(s)}\right) \leq \operatorname{reg}\left(\left(I(G)^{s+1}: L_{l+1}^{(s)}\right),\right.
$$

and the theorem follows.

As a corollary to the above theorem, we get the following important result:

Corollary 5.3 If for all $s \geq 1$ and for all minimal monomial generators $m$ of $I(G)^{s}$, $\operatorname{reg}\left(I(G)^{s+1}: m\right) \leq 2$ and $\operatorname{reg}(I(G)) \leq 4$, then for all $s \geq 1, \operatorname{reg}\left(I(G)^{s+1}\right)=2 s+2$; as a consequence, $I(G)^{s+1}$ has a linear minimal free resolution.

Proof We observe that under the condition if $\operatorname{reg}\left(I(G)^{s}\right) \leq 2 s+2$, then $\operatorname{reg}\left(I(G)^{s+1}\right) \leq 2 s+2$ too. Now $\operatorname{reg}(I(G)) \leq 4$ implies $\operatorname{reg}\left(I(G)^{2}\right) \leq 4$. By induction, assume $\operatorname{reg} I(G)^{k} \leq 2 k$. As $2 k<2 k+2$, reg $I(G)^{k} \leq 2 k+2$. Hence, $\operatorname{reg} I(G)^{k+1} \leq 2 k+2$. This proves the corollary.

\section{Bounding the regularity: the results}

In this section, we give some new bounds on $\operatorname{reg}\left(I(G)^{s}\right)$ for certain classes of gap-free graphs $G$. The main idea is to carefully analyze the ideal $\left(I(G)^{s+1}: e_{1} \ldots e_{s}\right)$ for an arbitrary $s$-fold product of edges, i.e., for $i \neq j, e_{i}=e_{j}$ is a possibility. Now, any $s$-fold product can be written as product of $s$ edges in various ways. In this section, we fix a presentation and work with respect to that. We first prove that these ideals are generated in degree two for any graph $G$.

Theorem 6.1 For any graph $G$ and for any $s$-fold product $e_{1} \ldots e_{s}$ of edges in $G$ (with the possibility of $e_{i}$ being same as $e_{j}$ as an edge for $\left.i \neq j\right)$, the ideal $\left(I(G)^{s+1}\right.$ : $\left.e_{1} \ldots e_{s}\right)$ is generated by monomials of degree two.

Proof We prove this using induction on $s$. For $s=0$, the result is clear as $(I(G)$ : $(1))=I(G)$, which is generated by monomials of degree two. Now, let us assume that the theorem is true till $s-1$. 
Let $m$ be a minimal monomial generator of $\left(I(G)^{s+1}: e_{1} \ldots e_{s}\right)$. Then, $e_{1} \ldots e_{s} m$ is divisible by an $(s+1)$-fold product of edges. By degree consideration, $m$ cannot have degree 1 . If $m$ has degree greater than or equal to 3 , then again by a degree consideration for some $i, e_{i}=p q$ such that $e_{1} \ldots e_{i-1} q e_{i+1} \ldots e_{s} m$ is divisible by an $(s+1)$-fold product of edges. Without loss of generality, we may assume $e_{1}=p q$ and there is an $(s+1)$-fold product $f_{1} \ldots f_{s+1}$ such that $f_{1} \ldots f_{s+1} \mid q e_{2} \ldots e_{s} m$.

If $q \mid f_{1} \ldots f_{s+1}$, without loss of generality, we may assume $f_{1}=p^{\prime} q$. So, $p^{\prime} q f_{2} \ldots f_{s+1} \mid q e_{2} \ldots e_{s} m$. Hence, $f_{2} \ldots f_{s+1} \mid e_{2} \ldots e_{s} m$. If $q$ does not divide $f_{1} \ldots$ $f_{s+1}$, then $f_{1} \ldots f_{s+1} \mid e_{2} \ldots e_{s} m$, and hence $f_{2} \ldots f_{s+1} \mid e_{2} \ldots e_{s} m$. In both cases, $m \in$ $\left(I(G)^{s}: e_{2} \ldots e_{s}\right)$.

Now $\left(I(G)^{s}: e_{2} \ldots e_{s}\right) \subset\left(I(G)^{s+1}: e_{1} \ldots e_{s}\right)$ and $m$ is a minimal monomial generator of $\left(I(G)^{s+1}: e_{1} \ldots e_{s}\right)$. So $m$ has to be a minimal monomial generator of $\left(I(G)^{s}: e_{2} \ldots e_{s}\right)$. Hence, by induction, $m$ has degree two, which is a contradiction to the assumption that $m$ has degree greater than or equal to three. Hence, $m$ has to have degree two.

To analyze the generators of $\left(I(G)^{s+1}: e_{1} \ldots e_{s}\right)$, we introduce the notion of even-connectedness with respect to $s$-fold products.

Definition 6.2 Two vertices $u$ and $v$ ( $u$ may be equal to $v$ ) are said to be even-connected with respect to an $s$-fold product $e_{1} \ldots e_{s}$ if there is a path $p_{0} p_{1} \ldots p_{2 k+1}, k \geq 1$ in $G$ such that

1. $p_{0}=u, p_{2 k+1}=v$.

2. For all $0 \leq l \leq k-1, p_{2 l+1} p_{2 l+2}=e_{i}$ for some $i$.

3. For all $i$,

$$
\left|\left\{l \geq 0 \mid p_{2 l+1} p_{2 l+2}=e_{i}\right\}\right| \leq\left|\left\{j \mid e_{j}=e_{i}\right\}\right|
$$

4. For all $0 \leq r \leq 2 k, p_{r} p_{r+1}$ is an edge in $G$.

If these properties are satisfied, then $p_{0}, \ldots, p_{2 k+1}$ is said to be an even-connection between $u$ and $v$ with respect to $e_{1} \ldots e_{s}$.

Example 6.3 Let $I(G)=(x y, x u, y v, y w, w z, z v)$ and $e_{1}=x y, e_{2}=w z$, then $u, x, y, w, z, v$ is an even-connection between $u$ and $v$ with respect to $e_{1} e_{2}$.

The following observation is an immediate consequence of the definition:

Observation 6.4 If $u=p_{0}, \ldots, p_{2 k+1}=v$ is an even-connection with respect to some $s$-fold product $e_{1} \ldots e_{s}$, then for any $j^{\prime} \geq j \geq 0$, any neighbor $x$ of $p_{2 j+1}$ and any neighbor y of $p_{2 j^{\prime}+2}$ are even-connected with respect to $e_{1} \ldots e_{s}$.

The next theorem also easily follows from the definition.

Theorem 6.5 If $u=p_{0}, \ldots, p_{2 k+1}=v$ is an even-connection with respect to some $s$-fold product $e_{1} \ldots e_{s}$, then $u v \in\left(I(G)^{s+1}: e_{1} \ldots e_{S}\right)$. 
Proof By conditions 2 and 3 of the definition, $e_{1} \ldots e_{s}=p_{1} \ldots p_{2 k} \cdot e_{j_{1}} \ldots e_{j_{s-k}}$, for some $\left\{j_{1}, j_{2}, \ldots, j_{s-k}\right\} \subset\{1, \ldots, s\}$ and by conditions 1 and $4, u p_{1} \ldots p_{2 k} v$ is a $(k+1)$-fold product of edges in $G$. Hence, $u v e_{1} \ldots e_{s}$ is an $(s+1)$-fold product of edges in $G$ and the result follows.

Although we fix a representation for all $s$-fold product and work with respect to that representation, it is worth noting that our definition of even-connectedness is independent of the representation we choose in the following sense:

Theorem 6.6 If $f_{1} \ldots f_{s}=e_{1} \ldots e_{s}$ are two different representations of same $s$-fold product as product of edges and $u$ and $v$ are even-connected with respect to $e_{1} \ldots e_{s}$, then $u$ and $v$ are even-connected with respect to $f_{1} \ldots f_{s}$.

Proof Let $u=p_{0}, \ldots, p_{2 k+1}=v$ be an even-connection between $u$ and $v$ with respect to $e_{1} \ldots e_{s}$. We shall construct an even-connection $q_{0}, \ldots, q_{2 r+1}$ between $u$ and $v$ with respect to $f_{1} \ldots f_{s}$.

Let $i$ be minimal such that $p_{2 i+1} p_{2 i+2}$ is not equal to any edge $f_{1}, \ldots, f_{s}$. Let $q_{0}=$ $p_{0}, \ldots, q_{2 i+1}=p_{2 i+1}$. We have $\left(u p_{1}\right)\left(p_{2} p_{3}\right) \ldots\left(p_{2 k} v\right) e_{t_{1}} \ldots e_{t_{s-k}}=(u v) f_{1} \ldots f_{s}$. Then $p_{2 i+1}\left(p_{2 i+2} p_{2 i+3}\right) \ldots\left(p_{2 k} v\right) e_{t_{1}} \ldots e_{t_{s-k}}=v f_{j_{1}} \ldots f_{j_{s-i}}$. If $v=p_{2 i+1}$, then we are done. Otherwise, $p_{2 i+1}$ divides one of the $f \mathrm{~s}$; without loss of generality let $f_{j_{1}}=$ $p_{2 i+1} q_{2 i+2}$. If $v q_{2 i+2}$ is an edge in $G$, then we are done by taking $q_{2 i+3}=v$. Otherwise, we have $v q_{2 i+2} f_{j_{2}} \ldots f_{s-i}$ is an $(s-i)$-fold product of edges $g_{1} \ldots g_{s-i}$, where without loss of generality $g_{1}=q_{2 i+2} q_{2 i+3}$ and $f_{j_{2}}=q_{2 i+3} q_{2 i+4}$. After selecting (without loss of generality) $g_{l}=q_{2 i+2 l} q_{2 i+2 l+1}$ and $f_{j_{l+1}}=q_{2 i+2 l+1} q_{2 i+2 l+2}$, we select $q_{2 i+2 l+3}$ inductively. If $v q_{2 i+2 l+2}$ is an edge in $G$, then we are done by choosing $q_{2 i+2 l+3}=v$. Otherwise, $g_{l+1} \ldots g_{s-i}=v q_{2 i+2 l+2} f_{j_{l+2}} \ldots f_{j_{s-i}}$. If $v$ is connected to $q_{2 i+2 l+2 k}$ for some $k$ in $G$ then we are done by choosing $q_{2 i+2 l+2 k+1}=v$. If not, then $g_{1} \ldots g_{s-i}=v g_{1} g_{2} \ldots g_{s-i-1} q_{2 i+2 s-2}$; but this will force $g_{s-i}=q_{2 i+2 s-2} v$, contradicting the fact that $v$ is not connected to $q_{2 i+2 l+2 k}$ for any $k$.

The conditions 1, 2, 4 of the definition are automatically satisfied by our construction. Condition 3 is satisfied because each $q_{2 i+1} q_{2 i+2}$ is $f_{r_{i}}$ for some integer $r_{i}$ and $q_{2 i+3} q_{2 i+4}$ is some $f_{r_{i+1}}$ where $r_{i+1} \notin\left\{r_{1}, \ldots, r_{i}\right\}$.

We now observe that all edges of $G$ belong to $\left(I(G)^{s+1}: e_{1} \ldots e_{s}\right.$ ). If $u v$ ( $u$ may be equal to $v$ ) belongs to $\left(I(G)^{s+1}: e_{1} \ldots e_{s}\right)$ and $u v$ is not an edge, then we prove that $u$ and $v$ have to be even-connected with respect to the $s$-fold product $e_{1} \ldots e_{s}$. The conditions 1, 2, 3, 4 are satisfied by the way of construction.

Theorem 6.7 Every generator $u v$ ( $u$ may be equal to $v$ ) of $\left(I(G)^{s+1}: e_{1} \ldots e_{s}\right.$ ) is either an edge of $G$ or even-connected with respect to $e_{1} \ldots e_{s}$, for $s \geq 1$.

Proof Suppose $u v$ is not an edge, and $u$ and $v$ are not even-connected. Now uve $_{1} \ldots e_{s}=f_{0} \ldots f_{s}$ is an $(s+1)$-fold product of edges, where $f_{0}=u p_{0}$ such that there is an edge $e_{i_{0}}=p_{0} q_{1}, 1 \leq i_{0} \leq s$. After selecting $f_{j}=q_{j} p_{j}$ and $e_{i_{j}}=p_{j} q_{j+1}, 1 \leq i_{j} \leq s$ and all $i_{j}$ are different, we select $f_{j+1}$ and $e_{i_{j+1}}$ inductively. $q_{j+1}$ is part of an edge $q_{j+1} p_{j+1}$ in the $(s+1)$-fold product $f_{0} \ldots f_{s}$. We choose $f_{j+1}=q_{j+1} p_{j+1}$. Now as $u$ and $v$ are not even-connected, $p_{j+1}$ is not $v$. So it is part of an edge amongst the remaining $e_{i}$ s. So, there exists $e_{i_{j+1}}=p_{j+1} q_{j+2}$, 
$i_{j+1} \in\{1, \ldots, s\} \backslash\left\{i_{1} \ldots i_{j}\right\}$. Now, as $u$ and $v$ are not even-connected, $v \neq p_{k}$ for any $k$. We observe $f_{0} \ldots f_{s}=u\left(p_{0} q_{1}\right)\left(p_{1} q_{2}\right) \ldots\left(p_{s-1} q_{s}\right) p_{s}=u v e_{1} \ldots e_{s}$. By construction, $\left(p_{0} q_{1}\right)\left(p_{1} q_{2}\right) \ldots\left(p_{s-1} q_{s}\right)=e_{1} \ldots e_{s}$. This forces $p_{s}=v$, which is a contradiction.

Example 6.8 Let $I(G)=(x y, x u, x v, x z, y z, y w)$. Then $\left(I(G)^{2}: x y\right)=I(G)+$ $\left(z^{2}, u z, v z, w z, u w, v w\right)$. Here, $z$ is even-connected to itself and $u, v, w$ with respect to $x y$; also $u, w$ and $v, w$ are even-connected with respect to $x y$.

We observe that $\left(I(G)^{s+1}: e_{1} \ldots e_{s}\right)$ need not be square free as there is a possibility that some vertex $u$ is even-connected to itself with respect to $e_{1} \ldots e_{s}$. So we polarize $\left(I(G)^{s+1}: e_{1} \ldots e_{s}\right)$ to get a square-free quadratic monomial ideal (i.e., an edge ideal) $\left(I(G)^{s+1}: e_{1} \ldots e_{s}\right)^{\text {pol }}$. For details of polarization, we refer to [8], Sect. 3.2 of [10] and exercise 3.15 of [10]. Here, we just recall the definition and one theorem which states a quadratic monomial ideal and its polarization have same regularity.

Definition 6.9 For any quadratic monomial ideal $I$ in $K\left[x_{1}, \ldots, x_{n}\right], I^{\text {pol }}$ is a square-free quadratic monomial ideal in $K\left[x_{1}, \ldots, x_{n}, x_{1}^{\prime}, \ldots, x_{n}^{\prime}\right]$ where $I^{\text {pol }}=$ $\left\langle x_{i} x_{j}, x_{k} x_{k}^{\prime} \mid x_{i} x_{j} \in I, x_{k}^{2} \in I\right\rangle$.

The following theorem, which we state without proof, is a special case of Proposition 1.3.4 of [8], we also refer to Sect. 3.2 and Exercise 3.15 of [10].

Theorem 6.10 $\operatorname{reg}\left(I^{\mathrm{pol}}\right)=\operatorname{reg}(I)$.

Clearly by Theorems $6.1,6.5$, and $6.7,\left(I(G)^{s+1}: e_{1} \ldots e_{s}\right)^{\text {pol }}$ is an edge ideal with the same regularity as $\operatorname{reg}\left(I(G)^{s+1}: e_{1} \ldots e_{s}\right)$. We describe the graph associated to this edge ideal in the following Lemma:

Lemma 6.11 $\left(I(G)^{s+1}: e_{1} \ldots e_{S}\right)^{\text {pol }}$ is the edge ideal of a new graph $G^{\prime}$ which has

1. All vertices and edges of $G$.

2. Any two vertices $u, v, u \neq v$ of $G$ that are even-connected with respect to $e_{1} \ldots e_{s}$ are connected by an edge in $G^{\prime}$.

3. For every vertex $u$ which is even-connected to itself with respect to $e_{1} \ldots e_{s}$, there is a new vertex $u^{\prime}$ which is connected to $u$ by an edge and not connected to any other vertex (so $u u^{\prime}$ is a whisker).

Proof By Theorem 6.7, every generator $u v$ ( $u$ may be equal to $v$ ) of $\left(I(G)^{s+1}\right.$ : $\left.e_{1} \ldots e_{s}\right)$ is either an edge of $G$ or even-connected with respect to $e_{1} \ldots e_{s}$, for $s \geq 1$. If it is an edge in $G$, then it satisfies condition 1 ; if it is an even-connection with $u \neq v$, then it satisfies condition 2; if it is an even-connection with $u=v$, then by definition of polarization, there will be a whisker $u^{\prime}$ on $u$ in $G^{\prime}$, and hence it will satisfy condition 3. Conversely, edges described by the conditions 1,2 and 3 belong to $G^{\prime}$ by Theorems 6.5 and 6.7 . 
Example 6.12 Let $G$ be the following graph:

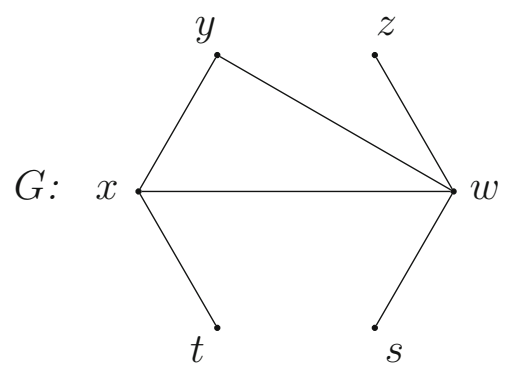

Then, the graph $G^{\prime}$ associated to $\left(I(G)^{2}: x w\right)^{\text {pol }}$ is the following:

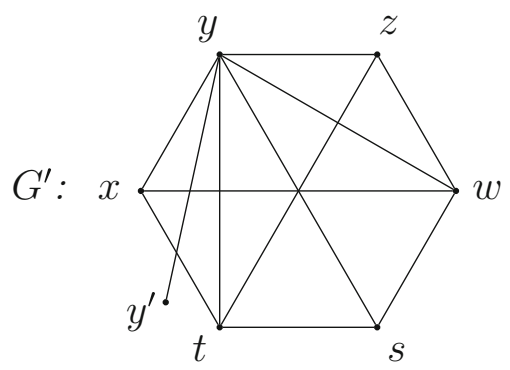

Next, we prove several lemmas that will be useful to get our main results.

Lemma 6.13 Suppose $u=p_{0}, \ldots, p_{2 k+1}=v$ is an even-connection between $u$ and $v$ and $z=q_{0}, \ldots, q_{2 l+1}=w$ is an even-connection between $z$ and $w$, both with respect to $e_{1} \ldots e_{s}$. If for some $i$ and $j, p_{2 i+1} p_{2 i+2}$ and $q_{2 j+1} q_{2 j+2}$ has a common vertex in $G$, then $u$ is even-connected to either $z$ or $w$ with respect to $e_{1} \ldots e_{s}$, and $v$ is even-connected to either $z$ or $w$ with respect to $e_{1} \ldots e_{s}$.

Proof We prove it for $u$, and the proof for $v$ follows by symmetry. Let $i$ be the smallest integer such that there is $j$ with the required property. If $p_{2 i+1}=q_{2 j+1}$, then $u=p_{0}, \ldots, p_{2 i+1}=q_{2 j+1}, q_{2 j+2}, q_{2 j+3}, \ldots, q_{2 l+1}=w$ gives an even-connection between $u$ and $w$ with respect to $e_{1} \ldots e_{s}$ (conditions 1,2 , and 4 are automatically satisfied, and condition 3 is satisfied as $i$ is the smallest integer such that there is a $j$ ). Similarly, if $p_{2 i+1}=q_{2 j+2}$, then $u=p_{0}, \ldots, p_{2 i+1}=q_{2 j+2}, q_{2 j+1}, q_{2 j}, \ldots, q_{0}=$ $z$ gives an even-connection between $u$ and $z$ with respect to $e_{1} \ldots e_{s}$; if $p_{2 i+1}$ is not equal to either $q_{2 j+1}$ or $q_{2 j+2}$ and $p_{2 j+2}=q_{2 j+1}$, then $u=p_{0}, \ldots, p_{2 i+1}, p_{2 j+2}=$ $q_{2 j+1}, q_{2 j+2}, q_{2 j+1}, q_{2 j}, \ldots, q_{0}=z$ gives an even-connection between $u$ and $z$ with respect to $e_{1} \ldots e_{s}$; if $p_{2 i+1}$ is not equal to either $q_{2 j+1}$ or $q_{2 j+2}$ and $p_{2 j+2}=q_{2 j+2}$, then $u=p_{0}, \ldots, p_{2 i+1}, p_{2 j+2}=q_{2 j+2}, q_{2 j+1}, q_{2 j+2}, \ldots, q_{2 l+1}=w$ gives an even-connection between $u$ and $w$ with respect to $e_{1} \ldots e_{s}$; in each of these cases, conditions 1,2, and 4 are satisfied automatically, and condition 3 is satisfied as $i$ is the smallest integer with the property. This covers all the cases.

The next two lemmas are results about gap-free graphs:

Lemma 6.14 If $G$ is gap free, then so is the graph $G^{\prime}$ associated to $\left(I(G)^{s+1}\right.$ : $\left.e_{1} \ldots e_{s}\right)^{\mathrm{pol}}$, for every $s$-fold product $e_{1} \ldots e_{s}$. 
Proof There are three possibilities of gap formation in $G^{\prime}$ :

1. Between two edges from $G$.

2. Between two edges that are not edges in $G$.

3. Between two edges where one of them is an edge in $G$ another is not.

No two edges in $G$ can form a gap in $G$ as $G$ is gap free. So they cannot form an edge in $G^{\prime}$ as in $G^{\prime}$, no edge of $G$ is being deleted.

For the second case, suppose $u v$ and $z w$ are even-connected with respect to $e_{1} \ldots e_{s}$, and neither $u v$ nor $z w$ is an edge in $G$. Without loss of generality, we may assume $\operatorname{gcd}(u v, z w)=1$ as there is no question of gap formation otherwise. Let $u=p_{0}, \ldots, p_{2 k+1}=v$ be an even-connection between $u, v$ with respect to $e_{1} \ldots e_{s}$, and let $z=q_{0}, \ldots, q_{2 l+1}=w$ be an even-connection between $z, w$ with respect to $e_{1} \ldots e_{s}$. In light of Lemma 6.13, we may assume for no $i, j, p_{i}=q_{j}$. If $u=q_{1}$, then $z u=z q_{1}$ is an edge in $G$ and if $z=p_{1}$, then $u z=u p_{1}$ is an edge in $G$, so there is nothing to prove. Otherwise, as $u p_{1}$ and $z q_{1}$ are edges in $G$ and $G$ is gap free, there are four possibilities:

a. $u$ is connected to $z$ in $G$, in which case $u v$ (or $u u^{\prime}$ in case $u=v$ ) and $z w$ (or $z z^{\prime}$ in case $z=w$ ) cannot form a gap, as in that case, $u z$ is an edge in $G^{\prime}$ too.

b. $p_{1}$ is connected to $z$, in which case $z, p_{1}, \ldots, p_{2 k+1}=v$ is an even-connection between $z$ and $v$ in $G$ so $z v$ is an edge in $G^{\prime}$; hence, $u v$ (or $u u^{\prime}$ if $u=v$ ) and $z w$ (or $z z^{\prime}$ if $z=w$ ) cannot form a gap.

c. $p_{1}$ is connected to $q_{1}$, in which case $v=p_{2 k+1}, p_{2 k}, \ldots, p_{1}, q_{1}, q_{2}, \ldots, q_{2 l+1}=$ $w$ gives an even-connection between $v$ and $w$, and $v w$ is an edge in $G^{\prime}$.

d. $q_{1}$ is connected to $u$, in which case $u, q_{1}, \ldots, q_{2 l+1}=w$ is an even-connection between $u$ and $w$ in $G$ so $u w$ is an edge in $G^{\prime}$; hence, $u v$ (or $u u^{\prime}$ if $u=v$ ) and $z w$ (or $z z^{\prime}$ if $z=w$ ) cannot form a gap.

In the third case, $u$ and $v$ are even-connected with respect to $e_{1} \ldots e_{s}$, and $z w$ is an edge in $G$, whereas $u v$ is not an edge in $G$. Like before, we may assume $\operatorname{gcd}(u v, z w)=$ 1. Let $u=p_{0}, \ldots, p_{2 k+1}=v$ be an even-connection between $u, v$ with respect to $e_{1} \ldots e_{s}$. If $z=p_{1}$, then $u z=u p_{1}$ is an edge in $G$ and if $w=p_{1}$, then $u w=u p_{1}$ is an edge in $G$, so there is nothing to prove in these cases. Otherwise, as $u p_{1}$ and $z w$ are edges in $G$ and $G$ is gap free, there are four choices:

a. $u$ is connected to $z$, in which case $u v$ (or $u u^{\prime}$ in case $u=v$ ) and $z w$ cannot form a gap as in that case, $u z$ is an edge $G^{\prime}$ too.

b. $p_{1}$ is connected to $z$, in which case $z, p_{1}, \ldots, p_{2 k+1}=v$ is an even-connection between $z$ and $v$ in $G$, so $z v$ is an edge in $G^{\prime}$; hence, $u v$ (or $u u^{\prime}$ if $u=v$ ) and $z w$ cannot form a gap.

c. $p_{1}$ is connected to $w$, in which case $v=p_{2 k+1}, p_{2 k}, \ldots, p_{1}, w$ is an evenconnection; hence, $u v$ and $z w$ can not form a gap.

d. $w$ is connected to $u$, in which case $u w$ is an edge in $G$, and hence in $G^{\prime}$.

This finishes the proof.

Lemma 6.15 Suppose $G$ is gap free. If $w_{1}, \ldots, w_{n}$ is an anticycle in the graph $G^{\prime}$ defined by $\left(I(G)^{s+1}: e_{1} \ldots e_{s}\right)$ for some $s \geq 1$ and for $n \geq 5$, then $w_{1}, \ldots, w_{n}$ is an anticycle in $G$. 
Proof First of all, whiskers on any vertex cannot be part of any anticycle of length $\geq 5$ as they only have degree 1 . Observe that it is enough to prove that for all $i$ and $j, w_{i}$ and $w_{i+j}$ are never even-connected with respect to $e_{1} \ldots e_{s}$. Suppose on the contrary such $i, j$ exists. Without loss of generality, we may choose $j$ to be minimal such that for some $i, w_{i}$ and $w_{i+j}$ are even-connected with respect to $e_{1} \ldots e_{s}$. Observe that $j \geq 2$ as $w_{i} w_{i+1}$ cannot be connected in an anticycle. Without loss of generality, we may further assume that $w_{1}$ and $w_{1+j}$ are even-connected with respect to $e_{1} \ldots e_{s}$ via $w_{1}=p_{0}, p_{1}, \ldots, p_{2 k+1}=w_{1+j}$. Now, observe that $w_{2+j}$ is not connected to $p_{1}$ by an edge in $G$ as that will force $w_{1+j}$ and $w_{2+j}$ to be connected in $G^{\prime}$ by Observation 6.4 leading to a contradiction. So, there exists a smallest $l \geq 0,2+j \leq n-l \leq n$ such that $w_{n-l}$ is not connected to $p_{1}$ by an edge in $G$. If $l=0$, then $w_{n}$ is not connected to $p_{1}$ by an edge in $G$ and if $l>0$, then $w_{n-l}$ is not connected to $p_{1}$ by an edge to $p_{1}$ in $G$, and $w_{n}, w_{n-1}, \ldots, w_{n-l+1}$ are connected to $p_{1}$ by an edge in $G$.

Next, we look at the edge $w_{2} w_{n-l}$ in $G^{\prime}$. If $w_{2}$ is connected to $p_{1}$ in $G$, then $w_{2}, p_{1}, \ldots, p_{2 k+1}=w_{1+j}$ will be an even-connection that will violate the minimality of $j$. If $w_{2}$ is connected to $p_{2}$ in $G$, then by Observation $6.4, w_{1} w_{2}$ has to be an edge in $G^{\prime}$, which will contradict the fact that $w_{1} \ldots w_{n}$ is an anticycle. We observe $w_{n-l}$ cannot be connected to $p_{1}$ by selection. If $w_{n-l}$ is connected to $p_{2}$ and $l=0$, then by Observation $6.4, w_{1}$ and $w_{n}$ have to be connected to each other in $G^{\prime}$. If $w_{n-l}$ is connected to $p_{2}$ and $l>0$, then by Observation $6.4, w_{n-l+1}$ and $w_{n-l}$ have to be connected to each other in $G^{\prime}$. Both cases lead to a contradiction as $w_{1} \ldots w_{n}$ is an anticycle, so $w_{2}$ and $w_{n-l}$ are not connected to each other in $G$, and neither of them is connected to $p_{1}$ or $p_{2}$ (and hence $w_{2}, w_{n-l}, p_{1}, p_{2}$ are four distinct vertices). As $p_{1} p_{2}$ is an edge in $G, w_{2} w_{n-l}$ cannot be an edge in $G$; otherwise, they will form a gap. So $w_{2}$ and $w_{n-l}$ are even-connected with respect to $e_{1} \ldots e_{s}$. Let $w_{2}=q_{0}, \ldots, q_{2 r+1}=w_{n-l}$ be an even-connection between $w_{2}$ and $w_{n-l}$ with respect to $e_{1} \ldots e_{s}$.

If for some $t_{1}, t_{2} \geq 0, p_{2 t_{1}+1} p_{2 t_{1}+2}$ and $q_{2 t_{2}+1} q_{2 t_{2}+2}$ are the same edges of $G$, then by Lemma $6.13, w_{2}$ has to be even-connected to either $w_{1}$ or $w_{1+j}$. The first case is not possible as $w_{1} \ldots w_{n}$ is an anticycle and the second case is not possible by the minimality of $j$. So for no $t_{1}, t_{2} \geq 0, p_{2 t_{1}+1} p_{2 t_{1}+2}$ and $q_{2 t_{2}+1} q_{2 t_{2}+2}$ are the same edges of $G$. So we look at $w_{n-l} q_{2 r}$ and $p_{1} p_{2}$. Observe that $p_{1}$ is not connected to $w_{n-l}$ because of the selection. If $w_{n-l}$ is connected to $p_{2}$ and $l=0$, then by Observation $6.4, w_{1}$ and $w_{n}$ have to be connected to each other in $G^{\prime}$. If $w_{n-l}$ is connected to $p_{2}$ and $l>0$, then by Observation 6.4, $w_{n-l+1}$ and $w_{n-l}$ have to be connected to each other in $G^{\prime}$. Both cases lead to a contradiction as $w_{1} \ldots w_{n}$ is an anticycle. So $p_{2}$ is not connected to $w_{n-l}$ in $G$. If $p_{1}$ is connected to $q_{2 r}$, then $w_{2}$ and $w_{1+j}$ will be evenconnected with respect to $e_{1} \ldots e_{s}$ violating the minimality of $j$. If $p_{2}$ is connected to $q_{2 r}$, then $w_{1}$ and $w_{2}$ will be even-connected and, hence, connected in $G^{\prime}$.

Hence, for no $i, j$ are $w_{i}$ and $w_{i+j}$ even-connected with respect to $e_{1} \ldots e_{s}$. So $w_{1} \ldots w_{n}$ is an anticycle in $G$.

Using this lemma, we get the following theorem of Herzog et al. [13, Theorem 1.2] as a corollary:

Theorem 6.16 If $I(G)$ has linear resolution, then for all $s \geq 2, I(G)^{s}$ has regularity $2 s$. In other words, $I(G)^{s}$ has a linear minimal free resolution. 
Proof As $I(G)$ has a linear resolution, it is gap free, and hence the polarizations of all $\left(I(G)^{s+1}: e_{1} \ldots e_{s}\right)$ are gap free by Lemma 6.14 , and any anticycle of length $\geq 5$ in the polarization of $\left(I(G)^{s+1}: e_{1} \ldots e_{S}\right)$ is an anticycle of $G$ by Lemma 6.15 . But as $I(G)$ has linear resolution, $G$ does not have any anticycle. By Theorem 2.12, $\operatorname{reg}\left(I(G)^{s+1}: e_{1} \ldots e_{s}\right)^{\mathrm{pol}}=2$ for all $e_{1} \ldots e_{s}$. Hence, $\operatorname{reg}\left(I(G)^{s+1}\right)=2 s+2$ by Theorems 1.2 and 6.10.

Next, we prove that for any gap free and cricket free graph $G$, and for all $s \geq 2$, $\operatorname{reg}\left(I(G)^{s}\right)=2 s$. This result is our main new result in this paper. This answers Question 1.1 partially. This also generalizes Nevo's result (Theorem 1.2 of [12]) that for any gap-free and claw-free graph $G, \operatorname{reg} I(G)^{2}=4$.

Theorem 6.17 For any gap free and cricket free graph $G$ and for all $s \geq 2$, $\operatorname{reg}\left(I(G)^{S}\right)=2 s$.

Proof In light of Theorem 2.12, Theorem 3.4, Corollary 5.3, Theorem 6.10, and Lemma 6.14, it is enough to show that the polarization of $\left(I(G)^{s+1}: e_{1} \ldots e_{s}\right)$ does not have any anticycle $w_{1} \ldots w_{n}$ for $n \geq 5, s \geq 1$, for every $s$-fold product $e_{1} \ldots e_{s}$.

Suppose $w_{1} \ldots w_{n}, n \geq 5$, is an anticycle in the polarization of $\left(I^{s+1}: e_{1} \ldots e_{s}\right)$ and $e_{1}=x y$. By Lemma 6.15, $w_{1} \ldots w_{n}$ is also an anticycle of $G$. Either $w_{1}$ or $w_{3}$ is a neighbor of $x$ or neighbor of $y$ else $w_{1} w_{3}$ and $e_{1}$ forms a gap in $G$, a contradiction. Without loss of generality, we may assume that $w_{1}$ is a neighbor of $x$. Now neither $w_{2}$ nor $w_{n}$ can be $x$ as they are not connected to $w_{1}$; also, neither of them is $y$ as if say $y=w_{2}$, then $w_{n} x y w_{1}$ is an even-connection; hence, $w_{1} w_{n}$ is an edge in $G^{\prime}$, a contradiction to the assumption on anticycle; similar thing happens if $y=w_{n}$. By Observation 6.4 every neighbor of $y$ is connected to every neighbor of $x$ in $G^{\prime}$. As neither $w_{1} w_{n}$, nor $w_{1} w_{2}$ is an edge in $G^{\prime}$, neither of $w_{2}$ and $w_{n}$ are neighbors of $y$ in $G$. So, one of them has to be neighbor of $x$, as $G$ is gap free. Again, without loss of generality, we may assume that $w_{2}$ is a neighbor of $x$. Next, we consider $w_{3} w_{n}$. As $w_{1}$ and $w_{2}$ are neighbors of $x$ and neither $w_{1} w_{n}$ nor $w_{2} w_{3}$ are edges in $G^{\prime}$, by Observation 6.4, neither $w_{3}$ nor $w_{n}$ can be neighbor of $y$. Neither $w_{3}$ nor $w_{n}$ can be $x$ as they are $w_{2} w_{3}$ and $w_{1} w_{n}$ are not edges in $G^{\prime}$. If $w_{3}=y$, as $w_{1} w_{3}$ is an edge in $G$, then $w_{1}$, being a neighbor of $y$, has to be connected to $w_{2}$, which is a neighbor of $x$ in $G^{\prime}$ by Observation 6.4. That will force $w_{1} w_{2}$ to be an edge in $G^{\prime}$, which is a contradiction. Similarly, if $w_{n}=y, w_{3}$ being a neighbor of $y$ has to be connected to $w_{2}$ in $G^{\prime}$ leading to a contradiction. Then, either $w_{3}$ or $w_{n}$ has to be a neighbor of $x$. Without loss of generality, we may assume that $w_{3}$ is a neighbor of $x$. Notice that $y$ is not connected to $w_{1}$ in $G$ as that will force $w_{2}$, a neighbor of $x$ to be connected to $w_{1}$ in $G^{\prime}$ leading to a contradiction. Hence, $\left\{y, w_{2}, x, w_{1}, w_{3}\right\}$ forms a cricket.

Next, we prove that for any gap-free graph $G$ with $\operatorname{reg}(I(G))=r$, the $\operatorname{reg}\left(I(G)^{S}\right)$ is bounded above by $2 s+r-1$. But to do that, we need a lemma about "longest" connections. Observe that if $G^{\prime}$ is the graph associated to the polarization of $\left(I(G)^{s+1}\right.$ : $e_{1} \ldots e_{s}$ ), for some $s$-fold product, and $u$ and $v$ are even-connected with respect to $u=p_{0}, \ldots, p_{2 k+1}=v$, then $u v$ is not only an edge in $G^{\prime}$ but also an edge in the graph $\left(G^{\prime}-\left\{y_{1}, \ldots, y_{l}\right\}\right)$ for any set of points $y_{1}, \ldots, y_{l}$ as long as $u, v \notin\left\{y_{1}, \ldots, y_{l}\right\}$. We further emphasize that some of the $p_{i}$ s can also belong to $\left\{y_{1}, \ldots, y_{l}\right\}$ as long as they are not same as $u$ or $v$. 
Lemma 6.18 Let $G^{\prime}$ be the graph associated to the polarization of $\left(I(G)^{s+1}\right.$ : $\left.e_{1} \ldots e_{s}\right)$ for some $s$-fold product. Let us assume that $u$ and $v$ are even-connected with respect to $u=p_{0}, \ldots, p_{2 k+1}=v$. Suppose for some set of vertices $\left\{y_{1}, \ldots, y_{l}\right\}$, we have $u, v \notin\left\{y_{1}, \ldots, y_{l}\right\}$. Let us also assume for any other even-connection $u^{\prime}=p_{0}^{\prime}, \ldots, p_{2 k^{\prime}+1}^{\prime}=v^{\prime}$ such that $u^{\prime}, v^{\prime} \notin\left\{y_{1}, \ldots, y_{l}\right\}$, we have $k^{\prime} \leq k$. Then, $\left(G^{\prime}-\left\{y_{1}, \ldots, y_{l}\right\}-\right.$ st $\left.u\right)$ is $G^{\prime \prime} \cup\{$ isolated whisker vertices $\}$, where $G^{\prime \prime}$ is a subgraph of $G$ obtained by deleting vertices.

Proof For the set of points $\left\{y_{1}, \ldots, y_{l}\right\}, u v$ is an edge in $\left(G^{\prime}-\left\{y_{1}, \ldots, y_{l}\right\}\right)$ such that $u, v \notin\left\{y_{1}, \ldots, y_{l}\right\}$ are even-connected with respect to $e_{1} \ldots e_{s}$ via $u=$ $p_{0}, p_{1}, p_{2}, \ldots, p_{2 k+1}=v$. We also have that $k$ is maximum over all such evenconnected edges in $\left(G^{\prime}-\left\{y_{1}, \ldots, y_{l}\right\}\right)$. Let $u^{\prime} v^{\prime}$ be any edge in $\left(G^{\prime}-\left\{y_{1}, \ldots, y_{l}\right\}\right)$ such that $u^{\prime}, v^{\prime} \notin\left\{y_{1}, \ldots, y_{l}\right\}$ and they are even-connected with respect to $e_{1} \ldots e_{s}$ via $u^{\prime}=x_{0}, x_{1}, x_{2}, \ldots, x_{2 k^{\prime}+1}=v^{\prime}$. If for any $j, j^{\prime}, p_{2 j+1} p_{2 j+2}$ and $x_{2 j^{\prime}+1} x_{2 j^{\prime}+2}$ form the same edge in $G$ then by Lemma 6.13, either $u^{\prime}$ or $v^{\prime}$ will be not a vertex in $\left(G^{\prime}-\left\{y_{1}, \ldots, y_{l}\right\}-s t u\right)$. Now observe, if for any $j, j^{\prime}, p_{2 j+1} p_{2 j+2}$ and $x_{2 j^{\prime}+1} x_{2 j^{\prime}+2}$ do not form same edge in $G$ then either $x_{1}$ or $x_{2}$ has to be connected to $p_{1}$ or $p_{2}$ to avoid $x_{1} x_{2}$ and $p_{1} p_{2}$ forming a gap. If any of them (for example $x_{1}$ ) is connected to $p_{1}$ in $G$ that will make $\left\{v^{\prime}=x_{2 k^{\prime}+1}, x_{2 k^{\prime}}, \ldots, x_{1}, p_{1}, \ldots, p_{2 k+1}\right\}$ a longer connection violating the maximality of $k$. A similar thing happens if $x_{2}$ is connected to $p_{1}$ in $G$. So either of them has to be connected to $p_{2}$. If $x_{1}$ is connected to $p_{2}$ in $G$, then $u$ is connected to $v^{\prime}$ in $G^{\prime}$ as $u, p_{1}, p_{2}, x_{1}, \ldots, x_{2 k^{\prime}+1}=v^{\prime}$ will be an even-connection. Similarly, if $x_{2}$ is connected to $p_{2}$, then $u$ is connected to $u^{\prime}$ in $G^{\prime}$ as $u, p_{1}, p_{2}, x_{2}, x_{1}, u^{\prime}$ will be an even-connection. In both the cases, either $u^{\prime}$ or $v^{\prime}$ will not be a vertex in $\left(G^{\prime}-\left\{y_{1}, \ldots, y_{l}\right\}-s t u\right)$. This proves that any edge in $\left(G^{\prime}-\left\{y_{1}, \ldots, y_{l}\right\}-\right.$ st $\left.u\right)$ is an edge in $G$. Hence, the Lemma follows.

Using Lemma 6.18, we prove the next theorem which guarantees that the gap between the regularity of powers of edge ideals of gap-free graphs and the regularity of monomial ideals generated in the same degree and having a linear resolution cannot be arbitrarily large.

Theorem 6.19 For any gap-free graph $G$ with $\operatorname{reg}(I(G))=r$ and any $s \geq 2$, the $\operatorname{reg}\left(I(G)^{s}\right)$ is bounded above by $2 s+r-1$.

Proof Let $G^{\prime}$ be the graph associated to the polarization of $\left(I(G)^{s+1}: e_{1} \ldots e_{s}\right)$. We have $\operatorname{reg}\left(G^{\prime}\right) \leq \max \left\{\operatorname{reg}\left(G^{\prime}-s t x\right)+1, \operatorname{reg}\left(G^{\prime}-x\right)\right\}$ by Lemma 3.2 for each vertex $x$. We choose $u_{1}$ and $v_{1}$ even-connected by $u_{1}=p_{0}, \ldots, p_{2 k_{1}+1}=v_{1}$ such that $k_{1}$ is maximum. By Lemma 6.18, $\left(G^{\prime}-s t u_{1}\right)$ is a subgraph of $G$ obtained by vertex deletion along with some isolated whisker vertices. As isolated vertices do not affect the regularity of edge ideal, $\operatorname{reg}\left(\left(G^{\prime}-s t u_{1}\right) \leq r\right.$ by Lemma 2.10 .

Next, we apply Lemma 3.2 on $\left(G^{\prime}-u_{1}\right)$, from which we delete a vertex $u_{2}$ which is even-connected to another vertex $v_{2}$ via $u_{2}=q_{0}, \ldots, q_{2 k_{2}+1}=v_{2}$ with $k_{2}$ maximum. Again, by Lemma 6.18, $\left(G^{\prime}-u_{1}-s t u_{2}\right)$ is a subgraph obtained from $G-u_{1}$ by deletion of vertices along with some whisker vertices. Hence, $\operatorname{reg}\left(G^{\prime}-u_{1}-s t u_{2}\right) \leq r$. We keep selecting $u_{1}, u_{2}, \ldots$ and apply Lemmas 3.2 and 6.18. As we are in a finite setup, for some $l,\left(G^{\prime}-u_{1}, \ldots, u_{l}\right)$ itself is a subgraph of $G$ obtained by repeated vertex 
deletion along with some isolated whisker vertices and $\operatorname{reg}\left(G^{\prime}\right) \leq r+1$. Therefore, by Theorem 1.2 and induction, the result follows.

Acknowledgments The author is very grateful to his advisor C. Huneke for constant support, valuable ideas and suggestions throughout the project. The author thanks G. Caviglia, A. De Stefani, J. Martin, J. Mermin and T. Vu for valuable discussions. The author also thanks the reviewers for suggesting many improvements and one anonymous reviewer for suggesting a simpler proof of Observation 4.10.

\section{References}

1. Dao, H., Huneke, C., Schweig, J.: Bounds on the regularity and projective dimension of ideals associated to graphs. J. Algebr. Combin. 38, 37-55 (2013)

2. Eisenbud, D., Green, M., Hulek, K., Popescu, S.: Restricting linear syzygies: algebra and geometry. Compos. Math. 141, 1460-1478 (2005)

3. Fröberg, R.: On Stanley-Reisner rings. In: Balcerzyk, S., et al. (eds.) Topics in Algebra, Part 2, vol. 26, pp. 57-70. Banach Center Publications, Reggio Calabria (1990)

4. Hà, H.T., Van Tuyl, A.: Splittable ideals and the resolutions of monomial ideals. J. Algebra 309, 405-425 (2007)

5. Ha, H.T., Van Tuyl, A.: Resolution of square-free monomial ideals via facet ideals: a survey. Contemp. Math. 448, 91-117 (2007)

6. Herzog, J., Hibi, T., Zheng, X.: Monomial ideals whose powers have a linear resolution. Math. Scand. 95, 23-32 (2004)

7. Hoefel, A.H., Whieldon, G.: Linear quotients of square of the edge ideal of the anticycle (2011). arXiv: $1106.2348 \mathrm{v} 2$

8. Kummini, M.: Homological invariants of monomial and binomial ideals. Thesis, University of Kansas (2008)

9. Kummini, M.: Regularity, depth and arithmetic rank of bipartite edge ideals. J. Algebr. Combin. 30, 429-445 (2009)

10. Miller, E., Sturmfels, B.: Combinatorial Commutative Algebra. Graduate Texts in Mathematics, vol. 227. Springer-Verlag, New York (2005)

11. Morey, S., Villarreal, R.: Edge Ideals: Algebraic and Combinatorial Properties. Progress in Commutative Algebra: Ring Theory, Homology and Decomposition. de Gruyter, Berlin (2012)

12. Nevo, E.: Regularity of edge ideals of $C_{4}$-free graphs via the topology of the lcm-lattice. J. Combin. Theory Ser. A 118, 491-501 (2011)

13. Nevo, E., Peeva, I.: $C_{4}$-free edge ideals. J. Algebr. Combin. 37, 243-248 (2013) 